\title{
A Newton-type Total Variation Diminishing Flow
}

\author{
Wolfgang Ring ${ }^{1}$ \\ Institute of Mathematics and Scientific Computing, Faculty of Natural Sciences, \\ University of Graz wolfgang.ring@uni-graz.at
}

\begin{abstract}
Summary. A new type of geometric flow is derived from variational principles as a steepest descent flow for the total variation functional with respect to a variable, Newton-like metric. The resulting flow is described by a coupled, non-linear system of differential equations. Written as one scalar evolution equation, the flow equation is non-local. Geometric properties of the flow are investigated, the relation to inverse scale space methods is discussed, and the question of appropriate boundary conditions is addressed. Numerical studies based on a finite element discretization are presented.
\end{abstract}

Key words: Geometric flow, Newton-type algorithm, mean curvature flow, image processing, inverse scale space

\section{Introduction}

\subsection{Geometric descent flow}

Geometric flow equations play an important role in image processing especially in image and surface smoothing and feature enhancement. In the image processing context, a gray-scale image is frequently represented by a function $\phi: U \subset \mathbb{R}^{n} \rightarrow \mathbb{R}$, with $n=2,3$ which assigns each point in the image domain $U$ a scalar value between 0 and 1 , the gray value of the image at this point. A time-dependent evolution equation of the form

$$
\phi_{t}=A(\phi) ; \quad \phi_{t=0}=\phi_{0}
$$

with an appropriate (possibly non-linear) operator $A$ can be used to process a given initial image $\phi_{0}$. The image $\phi_{0}$ then acts as the initial value for a continuous family of images $\phi(\cdot, t): U \rightarrow \mathbb{R}$ satisfying (1) for $t \in[0, T]$. It is usually the goal to define the flow equation in such a way that certain desirable features emerge in $\phi(\cdot, t)$ with passing evolution time. Various flows have been designed to reduce noise, to strengthen existing edges in the initial image, or 
to identify objects of a certain scale. Of special importance is the extraction or enhancement of geometrical features, i.e. properties which depend on the geometry of level-sets of the image function. Equation (1) is said to define a geometric flow if the family of level-sets

$$
\Gamma_{z}(t)=\{\mathbf{x} \in U: \phi(\mathbf{x}, t)=z\}
$$

for fixed $z \in \mathbb{R}$ depend on the initial data $\phi_{0}$ only via its $z$-level-set $\Gamma_{z}(0)=$ $\left\{\mathbf{x} \in U: \phi_{0}(\mathbf{x})=z\right\}$.

Examples of geometrical flows include mean curvature flow, total variation flow, and Willmore flow along with their various (anisotropic, scale-invariant, multi-channel etc.) versions. See e.g. [17, 24, 12], [28, 31], and [15] and the references therein for more information. All these examples are geometric flows which can be derived from variational principles of the form

$$
\min _{\phi} \int_{z \in \mathbb{R}} \int_{\Gamma_{z}} f_{z}(\mathbf{x}) d S(\mathbf{x}) d z
$$

where $\Gamma_{z}$ is the $z$-level-set of $\phi, d S$ denotes the surface measure on $\Gamma_{z}$ and $f_{z}: \Gamma_{z} \rightarrow \mathbb{R}$ is a function which depends only on intrinsic (geometrical) properties of $\Gamma_{z}$. If we choose $f_{z}=1$, mean curvature flow and total variation flow can be derived as different descent flows for the cost functional (2). (See Subsection 1.2). For the choice $f_{z}=\kappa^{2}$, where $\kappa$ is the mean curvature of the level-set $\Gamma_{z}$, Willmore flow is obtained. Using the co-area formula [16, Thm. 3.4.2] the functional (2) can be rewritten as

$$
\min _{\phi} J(\phi) \quad \text { with } J(\phi)=\int_{U} f(\mathbf{x}, \phi)|\nabla \phi| d \mathbf{x}
$$

and $f(\mathbf{x}, \phi)=f_{z}(\mathbf{x})$ for $\mathbf{x} \in \Gamma_{z}$. In this reformulation, the dependence of $f_{z}$ on geometrical properties of $\Gamma_{z}$ must be rewritten in terms of differential operators acting on the level-set function $\phi$. For (3) standard variational techniques can be applied to obtain a descent flow of the form (1).

Usually, finding the global minimum of (3) is not interesting (frequently the global minimum is attained for $\phi=0)$. One is rather interested in the transition from the initial image function $\phi_{0}$ to an advanced (steady) state $\phi_{\infty}$. In practise, the evolution of $\phi$ is stopped at an appropriate time $T$ for which the desired image properties are obtained. The descent flow is not uniquely determined by the cost functional (3). Assume, we have chosen a Banach space $X$ with $\phi \in X$ and we denote its dual space by $X^{\prime}$. The usual procedure is to differentiate the functional $J$ with respect to $\phi$ to get a first order approximation of the form

$$
J^{\prime}(\phi)(\psi)=\langle F, \psi\rangle_{X^{\prime}, X}
$$

and to choose a descent direction $\psi_{\text {desc }} \in X$ as the dual element to the negative derivative $-F \in X^{\prime}$. A dual element to $-F$ is defined by the properties $\langle F, \psi\rangle_{X^{\prime}, X}=-\|F\|_{X^{\prime}}$ and $\|\psi\|_{X}=1$. It is uniquely determined by $F$ if the 
space $X$ is reflexive and strictly convex [3, Thm. 1.2]. Obviously, the choice of $\psi_{\text {desc }}$ does depend not only on $F$ but also on the chosen function space $X$, especially on its norm $\|\cdot\|_{X}$ and the duality pairing $\langle\cdot, \cdot\rangle_{X^{\prime}, X}$. Note that equivalent norms do not yield identical dual elements.

Most constructions of descent flows for geometric functionals use a variant of an $L^{2}$-type norm to establish a connection between derivative and descent direction. In this paper we define and investigate an alternative norm for the construction of a descent direction with respect to the functional (2) for the special case $f_{z}=1$. This norm is defined using a positive definite approximation of the Hessian of the cost functional. This puts our approach within the class of Newton-type techniques. Moreover, the norm depends on the current level-set function $\phi$, thus we have a variable norm (variable metric) with every $t \in[0, T]$. Doing so, we mimic Newton's method from nonlinear optimization which aims at finding a descent direction pointing towards the minimum of a second-order approximation of the cost functional at every step of the algorithm (see [26] for details). It turns out that the operator on the right-hand side of the resulting flow equation (1) is non-local and the speed of propagation of level-sets depends on global properties such as their overall surface measure. The flow has certain similarities with the recently suggested inverse scale space approach for image smoothing. In the following, we present a derivation of the flow equation and a discussion of some of its geometric properties. We give numerical examples to investigate the behavior of the flow on individual level-sets and on the collection of all level-sets of a given image. We evaluation the dependence of the flow on certain parameters in the model and show that our approach is capable of interpolating between mean curvature flow and shrinking of shapes with constant speed.

\subsection{Mean Curvature and Bounded Variation Flows}

We choose the following notational convention: points in $\mathbb{R}^{n}$ as well as (tangential) vectors will be denoted by boldface letters e.g. $\mathbf{x}$ and $\mathbf{v}$. A vector $\mathbf{v}$ is always a (contravariant) column vector

$$
\mathbf{v}=\left(\begin{array}{c}
v_{1} \\
\vdots \\
v_{n}
\end{array}\right)
$$

The corresponding (covariant) row vector is denoted by $\mathbf{v}^{t}$. The gradient $\nabla f$ of a function $f: \Omega \subset \mathbb{R}^{n} \rightarrow \mathbb{R}$ is always a row vector. The scalar product of vectors $\mathbf{v}$ and $\mathbf{w}$ in $\mathbb{R}^{n}$ is denoted by $\langle\mathbf{v}, \mathbf{w}\rangle$ and sometimes also by $\mathbf{v}^{t} \cdot \mathbf{w}$ if this notation makes the presentation more transparent. We write the tensor product of two vectors $\mathbf{v}$ and $\mathbf{w}$ as $\mathbf{v} \otimes \mathbf{w}=\mathbf{v} \cdot \mathbf{w}^{t}=\left(v_{i} w_{j}\right)_{i, j=1}^{n}$.

The starting point for our considerations is the geometric surface area functional 


$$
J(\Gamma)=\int_{\Gamma} 1 d S=|\Gamma|
$$

where $\Gamma \subset \mathbb{R}^{n}$ is an $(n-1)$-dimensional subset (i.e. its $(n-1)$-dimensional Hausdorff measure is finite) and $d S$ denotes the integration with respect to the $(n-1)$-dimensional Hausdorff measure on $\Gamma$. We are specifically interested in the situation where $\Gamma=\Gamma_{z}$ is the $z$-level set of a function $\phi: \mathbb{R}^{n} \rightarrow \mathbb{R}$, i.e.

$$
\Gamma_{z}=\left\{\mathbf{x} \in \mathbb{R}^{n}: \phi(\mathbf{x})=z\right\}
$$

with some constant $z \in \mathbb{R}$. Usually we assume that $\Gamma=\partial \Omega$ with a bounded open set $\Omega=\mathbb{R}^{n}$. In the context of the level-set formulation (5) we assume that $\Gamma_{z}=\partial \Omega_{z}$ with $\Omega_{z}=\left\{\mathbf{x} \in \mathbb{R}^{n}: \phi(\mathbf{x})<z\right\}$.

We are interested in area diminishing flows i.e. smoothly varying families of surfaces $\{\Gamma(t): t \geq 0\}$ for which $|\Gamma(t)|$ is decreasing with increasing $t$. An obvious possibility to construct an area diminishing flow is to calculate the derivative of (4) with respect to $\Gamma$ and to propagate $\Gamma$ in a direction for which the directional derivative is negative. It is well known that the derivative of the area functional with respect to the geometric variable $\Gamma$ is given as

$$
d J(\Gamma ; \mathbf{v})=\int_{\Gamma} \kappa\langle\mathbf{v}, \mathbf{n}\rangle d S
$$

where $\mathbf{n}$ is the unit exterior normal vector to $\Gamma, \kappa$ denotes the mean curvature of $\Gamma$ and $\mathbf{v}: \mathbb{R}^{n} \rightarrow \mathbb{R}^{n}$ is the direction of perturbation of $\Gamma$. See for example [29, p. 116, eq. (2.174)] [13, p. 356, eq. (4.21) ], [30] for a parameter free derivation of the result, see e.g. [6], [32] for arguments using parametric curves and [2] for a very instructive comparison of the two approaches. To define a derivative it is necessary to specify the admissible perturbations of the independent variable. In the case of the geometric functional (6) we consider perturbations which are (in first order) of the form

$$
\Gamma^{h}=\left\{\mathbf{x}^{h}=\mathbf{x}+h \mathbf{v}(\mathbf{x}): \mathbf{x} \in \Gamma\right\} .
$$

With this (6) is obtained as the directional derivative

$$
d J(\Gamma ; \mathbf{v})=\lim _{h \rightarrow 0} \frac{1}{h}\left(J\left(\Gamma^{h}\right)-J(\Gamma)\right) .
$$

Suppose we choose $\mathbf{v}=-\kappa \mathbf{n}$ as direction of propagation. Of course, this choice is motivated by the fact that the directional derivative (6) is always non-positive in this direction. The corresponding area diminishing flow is the solution to the partial differential equation

$$
\frac{\partial \Gamma}{\partial t}=-\kappa \mathbf{n}
$$

Equation (7) is called the geometric heat equation and is extensively investigated in the literature (see [5] and the numerous references cited therein). 
Using Osher's and Sethian's idea [27], the propagation of the surface $\Gamma(t)$ can equivalently be expressed by a propagation law for a (now timedependent) level set function $\phi(t, \mathbf{x})$. In this context the family of surfaces $\Gamma(t)$ is implicitly given as $\Gamma(t)=\Gamma_{0}(t)=\left\{\mathbf{x} \in \mathbb{R}^{n}: \phi(t, \mathbf{x})=0\right\}$. The propagation of $\Gamma(t)$ with velocity given by $\mathbf{v}=F \mathbf{n}$ is translated into a propagation law for the level-set function:

$$
\phi_{t}+F|\nabla \phi|=0 .
$$

Setting $F=-\kappa$ in the level set equation (8) and noting that normal vector and curvature can be expressed as

$$
\mathbf{n}^{t}=\frac{\nabla \phi}{|\nabla \phi|} \text { and } \kappa=\operatorname{div}\left(\frac{\nabla \phi}{|\nabla \phi|}\right)
$$

the mean curvature flow equation

$$
\phi_{t}=|\nabla \phi| \operatorname{div}\left(\frac{\nabla \phi}{|\nabla \phi|}\right)
$$

is obtained. The zero-level set of $\phi$ plays no specific role in the implicit formulation (10). In fact, not only the zero-level set but all level sets of $\phi$ evolve according to the geometric heat equation (7). See $[17,18,19,20]$ and [8] for theoretical results and $[7,10,11]$ for numerical algorithms.

We present yet another derivation of (10) from a variational principle which is taken (at least in spirit) from [15]. For a Lipschitz continuous level set function $\phi: \mathbb{R}^{n} \rightarrow \mathbb{R}$ with compact support we consider the functional

$$
\tilde{J}(\phi)=\int_{z \in \mathbb{R}} \int_{\mathbf{x} \in \Gamma_{z}} 1 d S(\mathbf{x}) d z=\int_{\mathbf{x} \in \mathbb{R}^{n}}|\nabla \phi(\mathbf{x})| d \mathbf{x} .
$$

where we used the co-area formula [16, p. 112, Thm. 3.4.2] for the equivalence of the two expressions above. A sufficient requirement on the function $\phi$ for the co-area formula to hold is that $\phi$ is Lipschitz continuous. In the case that $|\nabla \phi|$ is not integrable on $\mathbb{R}^{n}$, equation (11) reproduces the trivial identity $\infty=\infty$. To avoid this type of degeneracy, we shall assume that $\phi$ is constant, and hence $\nabla \phi=0$ outside a ball of sufficiently large radius. Alternatively one can restrict the domain of integration to an arbitrary measurable subset $A \subset \mathbb{R}^{n}$ and work with the following form of the co-area formula:

$$
\int_{z \in \mathbb{R}} \int_{\mathbf{x} \in \Gamma_{z} \cap A} 1 d S(\mathbf{x}) d z=\int_{\mathbf{x} \in A}|\nabla \phi(\mathbf{x})| d \mathbf{x} .
$$

From the left-hand side of (11) if follows that $\tilde{J}$ simultaneously measures the areas of all level sets of the function $\phi$ in an integrated (averaged) form.

We now construct a flow for the level set function $\phi$ which diminishes the functional (11). The following calculations are formal i.e. without the 
necessary specification of the respective function spaces. Also the singularity for $|\nabla \phi|=0$ is treated formally and $|\nabla \phi|$ is replaced by $\sqrt{|\nabla \phi|^{2}+\epsilon^{2}}$ in the denominator with small $\epsilon>0$ for the concrete numerical calculations. Differentiation of $\tilde{J}$ with respect to $\phi$ gives

$$
\tilde{J}^{\prime}(\phi) \psi=\frac{\partial \tilde{J}}{\partial \phi} \psi=\int_{\mathbb{R}^{n}} \frac{\langle\nabla \phi, \nabla \psi\rangle}{|\nabla \phi|} d \mathbf{x}
$$

for the derivative of $\tilde{J}$ with respect to $\phi$ in the direction $\psi$. We can find a direction of propagation $\psi_{\text {desc }}$ for an area diminishing flow by minimizing the predicted descent (13) with respect to $\psi$ under the norm constraint

$$
\|\psi\|_{\text {desc }}^{2}=1,
$$

where $\|\cdot\|_{\text {desc }}$ is an appropriate norm for the descent direction $\psi$. We want the propagation to be of level-set form i. e.

$$
\phi_{t}-\psi=\phi_{t}+F|\nabla \phi|=0,
$$

that is, we set $\psi=-F|\nabla \phi|$. This, and the first variant of the cost functional (11) motivates the choice of the norm for $\psi$ in (14) as the $L^{2}$-norm of the equivalent speed function $F$ on $\Gamma_{z}$ integrated over all $z \in \mathbb{R}$. More precisely we use $\|\psi\|_{\text {desc }}=\|\psi\|_{L^{2}, \phi}$ with

$$
\begin{aligned}
\|\psi\|_{L^{2}, \phi}^{2}=\int_{z \in \mathbb{R}} \int_{\Gamma_{z}} F^{2}(\mathbf{x}) d S(\mathbf{x}) d z= & \int_{\mathbf{x} \in \mathbb{R}^{n}} F^{2}(\mathbf{x})|\nabla \phi(\mathbf{x})| d \mathbf{x} \\
& =\int_{\mathbf{x} \in \mathbb{R}^{n}} \psi^{2}(\mathbf{x})|\nabla \phi(\mathbf{x})|^{-1} d \mathbf{x} .
\end{aligned}
$$

To derive the necessary optimality conditions for the minimization of (13) under the constraint (14) with norm given by (16) we introduce the Lagrange functional

$$
\mathcal{L}(\psi, \lambda)=\int_{\mathbb{R}^{n}} \frac{\langle\nabla \phi, \nabla \psi\rangle}{|\nabla \phi|} d \mathbf{x}+\lambda\left(\int_{\mathbb{R}^{n}} \psi^{2}|\nabla \phi|^{-1} d \mathbf{x}-1\right) .
$$

From the Karush-Kuhn-Tucker condition $\mathcal{L}_{\psi}=0$ we get

$$
\operatorname{div}\left(\frac{\nabla \phi}{|\nabla \phi|}\right)+2 \lambda \psi|\nabla \phi|^{-1}=0 .
$$

Consequently

$$
\psi_{\mathrm{desc}}=\frac{1}{2 \lambda}|\nabla \phi| \operatorname{div}\left(\frac{\nabla \phi}{|\nabla \phi|}\right),
$$

where $\lambda>0$ is chosen such that $\|\psi\|_{L^{2}, \phi}=1$. Inserting this in (15) we get (after a re-scaling of the time variable) the mean curvature flow equation (10). 
Suppose we had chosen a different norm $\|\cdot\|_{\text {desc }}$ as given in (16). Then the resulting optimality system has a different form and we arrive at a flow equation for $\phi$ which is different from (10) but still has the property to decrease the functional (11) although along a different trajectory. As a second possibility we choose $\|\cdot\|_{\text {desc }}=\|\cdot\|_{L^{2}}$ where

$$
\|\psi\|_{L^{2}}^{2}=\int_{\mathbb{R}^{n}} \psi^{2} d \mathbf{x}
$$

the usual (non-geometric) $L^{2}$-norm on $\mathbb{R}^{n}$. With this, the necessary optimality condition reads as

$$
\operatorname{div}\left(\frac{\nabla \phi}{|\nabla \phi|}\right)+2 \lambda \psi=0
$$

and hence

$$
\psi_{\mathrm{desc}}=\frac{1}{2 \lambda} \operatorname{div}\left(\frac{\nabla \phi}{|\nabla \phi|}\right) .
$$

The corresponding flow equation is the well investigated total variation flow

$$
\phi_{t}=\operatorname{div}\left(\frac{\nabla \phi}{|\nabla \phi|}\right)
$$

See e.g. $[28,31,14,1]$.

\subsection{Outline}

Our paper is concerned with the construction of a different class of norms $\|\cdot\|_{\text {desc }}$ for the determination of the descent direction. The norm in the new approach is derived from the second order approximation of the cost functional at the current argument $\phi$. Consequently, the norm varies along with the propagating level-set function. This is, by the way, also the case for the geometric $L^{2}$-norm (16). The approach can therefore be considered as variable metric or Newton-type equivalent to the first order, gradient-type flows (10) and (19). In the following we present a derivation of the flow equations, we investigate geometric properties of the flow, we discuss the relation of the approach to inverse scale space methods, and we report the results of our numerical and experimental investigations for the new approach. In Section 2 we construct a norm from the Hessian of the cost functional (11) and we derive the corresponding steepest descent flow. In section 3, theoretical arguments are presented which shed some light on the geometrical and qualitative behavior of the flow. Moreover, the relation to inverse scale space methods is discussed. Boundary conditions are introduced and an alternative formulation of the system of flow equations is derived. Section 4 contains numerical examples and parameter studies. The behavior of the flow on individual level-sets on the one hand, and simultaneously on all level-sets of an image on the other hand, is investigated. 


\section{A Newton-type Flow for the Minimization of the Area of Level-Sets}

We aim for the construction of an alternative evolution equation for the level set function $\phi$ which also has the property of decreasing the areas of level sets with increasing time. We do so by replacing the vector field $-\kappa \mathbf{n}$ in (7) by vector fields of the form $F \mathbf{n}$ where the scalar function $F$ is found as a steepest descent direction. The corresponding metrics are constructed from the second order derivatives of the area functionals (4) and (11) respectively.

Formal differentiation of (13) with respect to $\phi$ gives the second derivative of the functional (11) as the bilinear form

$$
\tilde{J}^{\prime \prime}(\phi)(\psi, \eta)=\int_{\mathbb{R}^{n}} \frac{1}{|\nabla \phi|}\left(\langle\nabla \psi, \nabla \eta\rangle-\frac{1}{|\nabla \phi|^{2}}\langle\nabla \phi, \nabla \psi\rangle\langle\nabla \phi, \nabla \eta\rangle\right) d \mathbf{x} .
$$

The classical Newton (variable metric) approach suggests to use $\|\psi\|_{\text {desc }}^{2}=$ $\tilde{J}^{\prime \prime}(\phi)(\psi, \psi)$ provided that the bilinear form on the right-hand side is positive definite. Since

$$
|\nabla \psi|^{2}-\left\langle\frac{\nabla \phi}{|\nabla \phi|}, \nabla \psi\right\rangle^{2} \geq|\nabla \psi|^{2}-\left|\frac{\nabla \phi}{|\nabla \phi|}\right|^{2}|\nabla \psi|^{2}=0
$$

we find that the bilinear form (20) is positive semi-definite. It is not positive definite since $\tilde{J}^{\prime \prime}(\phi)(\psi, \psi)=0$ for any $\psi$ which is constant on level sets of $\phi$. In fact, if we set $\psi=f(\phi)$ with an arbitrary (smooth enough) function $f: \mathbb{R} \rightarrow \mathbb{R}$, we obtain

$$
|\nabla \psi|^{2}-\left\langle\frac{\nabla \phi}{|\nabla \phi|}, \nabla \psi\right\rangle^{2}=f^{\prime}(\phi)^{2}|\nabla \phi|^{2}-\left(f^{\prime}(\phi)|\nabla \phi|\right)^{2}=0 .
$$

We use a positive definite approximation to the Hessian as metric for the descent direction thus putting our approach into the class of inexact Newton methods. More precisely we set $\|\psi\|_{\text {desc }}=\|\psi\|_{\mathrm{N}, \phi}$ (the subscript 'N' stands for a Newton-type metric and $\phi$ indicates the dependence of the variable metric on the current level-set function) with

$$
\|\psi\|_{\mathrm{N}, \phi}^{2}=\tilde{J}^{\prime \prime}(\phi)(\psi, \psi)+\alpha\|\psi\|_{L^{2}, \phi}^{2}
$$

with some parameter $\alpha>0$ and $\|\psi\|_{L^{2}, \phi}$ as given in (16). Alternatively, we shall also use

$$
\|\psi\|_{\mathrm{N} 1, \phi}^{2}=\tilde{J}^{\prime \prime}(\phi)(\psi, \psi)+\alpha\|\psi\|_{L^{2}}^{2},
$$

with $\|\psi\|_{L^{2}}$ defined in (18). As descent direction we choose the solution to the constrained minimization problem

$$
\begin{aligned}
& \min _{\psi} J^{\prime}(\phi) \psi \\
& \text { such that }\|\psi\|_{\mathrm{N}, \phi}^{2}=1 .
\end{aligned}
$$


The Lagrange functional for the constrained optimization problem (23) with the choice (21) for $\|\cdot\|_{\mathrm{N}, \phi}$ is given as

$$
\begin{aligned}
& \mathcal{L}_{\mathrm{N}, \phi}(\psi, \lambda)=\int_{\mathbb{R}^{n}} \frac{\langle\nabla \phi, \nabla \psi\rangle}{|\nabla \phi|} d \mathbf{x}+ \\
& \lambda\left(\int_{\mathbb{R}^{n}} \frac{1}{|\nabla \phi|}\left(|\nabla \psi|^{2}-\frac{1}{|\nabla \phi|^{2}}\langle\nabla \phi, \nabla \psi\rangle^{2}\right) d \mathbf{x}+\alpha \int_{\mathbb{R}^{n}} \frac{\psi^{2}}{|\nabla \phi|} d \mathbf{x}-1\right) .
\end{aligned}
$$

The necessary optimality condition $\partial_{\psi} \mathcal{L}_{\mathrm{N}, \phi}=0$ yields that the minimizer $\hat{\psi}$ to (23) and the optimal multiplier $\hat{\lambda}$ must satisfy

$$
\begin{array}{r}
\int_{\mathbb{R}^{n}} \frac{1}{|\nabla \phi|}\left(\langle\nabla \hat{\psi}, \nabla \eta\rangle-\frac{1}{|\nabla \phi|^{2}}\langle\nabla \phi, \nabla \hat{\psi}\rangle\langle\nabla \phi, \nabla \eta\rangle\right) d \mathbf{x}+\alpha \int_{\mathbb{R}^{n}} \frac{\hat{\psi} \eta}{|\nabla \phi|} d \mathbf{x} \\
=-\frac{1}{2 \hat{\lambda}} \int_{\mathbb{R}^{n}} \frac{\langle\nabla \phi, \nabla \eta\rangle}{|\nabla \phi|} d \mathbf{x}
\end{array}
$$

for all test functions $\eta \in \mathcal{D}\left(\mathbb{R}^{n}\right)$. If we had used the norm (22) instead of (21), the last term in (25) must be replaced by $\alpha \int_{\mathbb{R}^{n}} \hat{\psi} \eta d \mathbf{x}$. Again, the Lagrange multiplier can be chosen $\hat{\lambda}=\frac{1}{2}$ since any other choice of the multiplier only modifies the size of the descent direction $\hat{\psi}$ and any such modification can be compensated by a re-scaling of the time variable in (15).

We have, therefore, found a weak form of a second order (Newton type) descent flow for the cost functional (11) with respect to the metric (21) as the coupled system

$$
\begin{aligned}
& \int_{\mathbb{R}^{n}} \frac{1}{|\nabla \phi|}\left(\langle\nabla \psi, \nabla \eta\rangle-\left\langle\frac{\nabla \phi}{|\nabla \phi|}, \nabla \psi\right\rangle\left\langle\frac{\nabla \phi}{|\nabla \phi|}, \nabla \eta\right\rangle+\alpha \psi \eta\right) d \mathbf{x} \\
& =-\int_{\mathbb{R}^{n}} \frac{\langle\nabla \phi, \nabla \eta\rangle}{|\nabla \phi|} d \mathbf{x}, \\
& \phi_{t}=\psi \quad \text { on } \mathbb{R}^{n},
\end{aligned}
$$

for all test functions $\eta \in \mathcal{D}\left(\mathbb{R}^{n}\right)$. Here (and in the following) we have changed the notation from the specific solution $\hat{\psi}$ of (23) back to the generic $\psi$.

Next, we rewrite equation (26a) in strong form. Beforehand, we provide a few useful relations. With (9) we get for the derivative of the mapping $\mathbf{n}: \mathbb{R}^{n} \rightarrow \mathbb{R}^{n}$

$$
\left.D \mathbf{n}=\frac{1}{|\nabla \phi|}\left(D^{2} \phi-\mathbf{n} \cdot \mathbf{n}^{t} \cdot D^{2} \phi\right\rangle\right)=\frac{1}{|\nabla \phi|}(I-\mathbf{n} \otimes \mathbf{n}) \cdot D^{2} \phi .
$$

at all points where $\mathbf{n}$ is differentiable. Note that $D \mathbf{n}(\mathbf{x}) \in \mathbb{R}^{3 \times 3}$ in (27) is not the intrinsic shape operator (the Weingarten map) $D \mathbf{n}: T_{\mathbf{x}} \Gamma \rightarrow T_{\mathbf{x}} \Gamma$ as e.g. described in [25]. Especially, $D \mathbf{n}$ in our case is not symmetric, the asymmetry being related to the behavior of $D \mathbf{n}$ in normal direction. For later use, we make the following consideration. From $\mathbf{n}^{t} \cdot \mathbf{n}=1$ we get by differentiation 


$$
0=\nabla\left(\mathbf{n}^{t} \cdot \mathbf{n}\right)=2 \mathbf{n}^{t} \cdot D \mathbf{n}
$$

Note that, on the other hand, $D \mathbf{n} \cdot \mathbf{n} \neq 0$ for each (generic) situation where the normals to level sets of $\phi$ are not straight lines. This implies that $D \mathbf{n}$ is not symmetric in general. We shall also use the relation

$$
\nabla\left(\frac{1}{|\nabla \phi|}\right)=-\frac{1}{|\nabla \phi|^{2}} \mathbf{n}^{t} \cdot D^{2} \phi
$$

To find a strong formulation for equation (25) we use Green's formula on all terms $\nabla \eta$ in (25). We obtain

$$
-\operatorname{div}\left(\frac{1}{|\nabla \phi|}\left(\nabla \psi-\left\langle\frac{\nabla \phi}{|\nabla \phi|}, \nabla \psi\right\rangle \frac{\nabla \phi}{|\nabla \phi|}\right)\right)+\alpha \frac{\psi}{|\nabla \phi|}=\operatorname{div}\left(\frac{\nabla \phi}{|\nabla \phi|}\right) .
$$

We introduce the Hessian operator $H(\phi)$ as

$$
H(\phi) \cdot \psi=-\operatorname{div}\left(\frac{1}{|\nabla \phi|}\left(\nabla \psi-\left\langle\frac{\nabla \phi}{|\nabla \phi|}, \nabla \psi\right\rangle \frac{\nabla \phi}{|\nabla \phi|}\right)\right)+\alpha \frac{\psi}{|\nabla \phi|} .
$$

With this, (26) can be (formally) written as an evolution equation for $\phi$ :

$$
\phi_{t}=(H(\phi))^{-1}\left[\operatorname{div}\left(\frac{\nabla \phi}{|\nabla \phi|}\right)\right] .
$$

For fixed $\phi \in \mathcal{C}^{1}\left(\mathbb{R}^{n}\right)$ and if $|\nabla \phi|$ in the denominators is replaced by a strictly positive approximation, equation (30) is a proper, degenerate elliptic equation in the sense of viscosity solutions. It can be shown, using Perrons method for existence and a comparison principle for uniqueness that (30) has a unique, locally Lipschitz continuous viscosity solution $\psi$. See [9] for a comprehensive introduction to the theory of viscosity solutions for degenerate elliptic equation. So far, we can only proof existence and uniqueness for equation (30) but not for the coupled system (30) and (26b) (or for the integrated formulation (32)). A thorough theoretical investigation of (32) is going to be the content of future work. Note that the evolution equation (32) is non-local in the spacial variable $\mathbf{x}$ since $H^{-1}$ is a non-local operator.

We write (30) in a more geometric form. Resolving the div-operator and using the notation (9) yields for the first term on the left hand side of (30): 


$$
\begin{aligned}
& \frac{1}{|\nabla \phi|^{2}}\left\langle\mathbf{n}^{t} \cdot D^{2} \phi, \nabla \psi-\langle\mathbf{n}, \nabla \psi\rangle \mathbf{n}^{t}\right\rangle \\
& \quad-\frac{1}{|\nabla \phi|}\left(\Delta \psi-\mathbf{n}^{t} \cdot D^{2} \psi \cdot \mathbf{n}-\nabla \psi \cdot D \mathbf{n} \cdot \mathbf{n}-(\nabla \psi \cdot \mathbf{n}) \operatorname{div}(\mathbf{n})\right) \\
& =\frac{1}{|\nabla \phi|^{2}}\left(\nabla \psi \cdot(I-\mathbf{n} \otimes \mathbf{n}) \cdot D^{2} \phi \cdot \mathbf{n}\right) \\
& \quad-\frac{1}{|\nabla \phi|}\left(\Delta \psi-\mathbf{n}^{t} \cdot D^{2} \psi \cdot \mathbf{n}-(\nabla \psi \cdot \mathbf{n}) \operatorname{div}(\mathbf{n})-\right. \\
& \left.\quad \frac{1}{|\nabla \phi|}\left(\nabla \psi \cdot(I-\mathbf{n} \otimes \mathbf{n}) \cdot D^{2} \phi \cdot \mathbf{n}\right)\right) \\
& =\frac{2}{|\nabla \phi|^{2}}\left(\nabla \psi \cdot(I-\mathbf{n} \otimes \mathbf{n}) \cdot D^{2} \phi \cdot \mathbf{n}\right) \\
& \quad-\frac{1}{|\nabla \phi|}\left(\Delta \psi-\mathbf{n}^{t} \cdot D^{2} \psi \cdot \mathbf{n}-(\nabla \psi \cdot \mathbf{n}) \operatorname{div}(\mathbf{n})\right)
\end{aligned}
$$

Here we used the identities (29) and (27). With this, we obtain

$$
\frac{1}{|\nabla \phi|}\left(-\left(\Delta \psi-\mathbf{n}^{t} \cdot D^{2} \psi \cdot \mathbf{n}-(\nabla \psi \cdot \mathbf{n}) \operatorname{div}(\mathbf{n})\right)+2 \nabla \psi \cdot D \mathbf{n} \cdot \mathbf{n}+\alpha \psi\right)=\operatorname{div}(\mathbf{n})
$$

for an equivalent formulation to (30).

Yet another - in certain respects more compact — variant of the system (33) can be found if we use the scalar speed function $F$ as update direction instead of $\psi$. Setting $\psi=F|\nabla \phi|$ and using

$$
\frac{\nabla \psi}{|\nabla \phi|}=\nabla F+\frac{F}{|\nabla \phi|^{2}} \nabla \phi \cdot D^{2} \phi
$$

we obtain for $(30)$

$$
\begin{aligned}
& -\operatorname{div}\left(\nabla F+\frac{F}{|\nabla \phi|^{2}} \nabla \phi \cdot D^{2} \phi-\left\langle\mathbf{n}, \nabla F+\frac{F}{|\nabla \phi|^{2}} \nabla \phi \cdot D^{2} \phi\right\rangle \mathbf{n}^{t}\right)+\alpha F \\
& =-\operatorname{div}\left(\nabla F-\langle\nabla F, \mathbf{n}\rangle \mathbf{n}^{t}+\frac{F}{|\nabla \phi|} \mathbf{n}^{t} \cdot D^{2} \phi \cdot(I-\mathbf{n} \otimes \mathbf{n})\right)+\alpha F \\
& =-\operatorname{div}\left(\nabla F-\langle\nabla F, \mathbf{n}\rangle \mathbf{n}^{t}+F(D \mathbf{n} \cdot \mathbf{n})^{t}\right)+\alpha F \\
& =-\left(\Delta F-\mathbf{n}^{t} \cdot D^{2} F \cdot \mathbf{n}-\operatorname{div}(\mathbf{n}) \nabla F \cdot \mathbf{n}\right)+F(\alpha+\operatorname{div}(D \mathbf{n} \cdot \mathbf{n}))=\operatorname{div}(\mathbf{n}) .
\end{aligned}
$$

As an equation for $F$ the formulation (34) is somewhat less complicated than equation (33). However, the term $\operatorname{div}(D \mathbf{n} \cdot \mathbf{n})$ comprises third derivatives of $\phi$ and the coupled system (34) together with the level set equation $\phi_{t}+F|\nabla \phi|=$ 0 is more involved. We mostly deal with the system (33) in the subsequent considerations. 


\section{Geometric properties}

In this section we investigate geometric and qualitative properties of the flow (33). We will show that the flow is close to intrinsic in the sense that the speed $\psi$ depends only on geometric properties of the level-sets of the function $\phi$, on $D \mathbf{n}$ and on $|\nabla \phi|$. We also consider the similarities and differences to a flow with constant normal speed and to inverse scale space methods.

\subsection{Intrinsic Formulation}

We make a few comments on the terms in (33). Using the differential geometric notation (9) we get

$$
\Delta \psi-\mathbf{n}^{t} \cdot D^{2} \psi \cdot \mathbf{n}-(\nabla \psi \cdot \mathbf{n}) \operatorname{div}(\mathbf{n})=\Delta \psi-\frac{\partial^{2} \psi}{\partial n^{2}}-\kappa \frac{\partial \psi}{\partial n}=\Delta_{\Gamma} \psi
$$

where $\Delta_{\Gamma}$ denotes the Laplace-Beltrami operator with respect to the level sets of $\phi$ (see [29, Prop. 2.68, p. 94$]$ ). The differential operator $\Delta_{\Gamma}$ is intrinsic on level sets of $\phi$, i.e. $\Delta_{\Gamma} \psi(\mathbf{x})$ depends only on values of $\psi$ on the level set $\{\mathbf{y}: \phi(\mathbf{y})=\phi(\mathbf{x})\}$.

The term in $\nabla \psi \cdot D \mathbf{n} \cdot \mathbf{n}(33)$ is in fact the directional derivative of $\psi$ with direction given by $D \mathbf{n} \cdot \mathbf{n}$. Due to $(28)$ we have $\mathbf{n}^{t} \cdot D \mathbf{n} \cdot \mathbf{n}=0$ hence, the normal component of the direction vanishes, i.e. $\nabla \psi \cdot D \mathbf{n} \cdot \mathbf{n}$ is a tangential derivative with with respect to the level set $\{\phi=$ const $\}$. It therefore follows that the differential equation (33) is intrinsic with on level sets of $\phi$ and can (or could if we wanted to resolve level sets) be solved independently for $\psi$ on each level set of $\phi$. The corresponding form of (33) is given by

$$
-\Delta_{\Gamma} \psi+2 \nabla \psi \cdot D \mathbf{n} \cdot \mathbf{n}+\alpha \psi=|\nabla \phi| \kappa \quad \text { on } \Gamma
$$

for each level-set $\Gamma=\Gamma_{z}=\{\phi=z\}$. Equation (35) is intrinsic for the variable $\psi$. The coefficients of (35), however, depend not only on the geometry of level sets. The terms $D \mathbf{n} \cdot \mathbf{n}$ on the left-hand side and $|\nabla \phi|$ on the right-hand side are not only on the geometry of the level-sets, but constitute a coupling between level-sets.

The nature of the non-intrinsic terms becomes more apparent in the formulation (34). We have

$$
\operatorname{div}(D \mathbf{n} \cdot \mathbf{n})=\operatorname{tr}\left(D \mathbf{n} \cdot D \mathbf{n}^{t}\right)+\langle\nabla \operatorname{div}(\mathbf{n}), \mathbf{n}\rangle=\sum_{i=1}^{n-1} \kappa_{i}^{2}+\partial_{\mathbf{n}} \kappa,
$$

where $\kappa_{i}$ denote the principal curvatures of $\Gamma$. With this, we arrive at the system

$$
\begin{aligned}
& -\Delta_{\Gamma} F+\left(\alpha+\sum_{i=1}^{n-1} \kappa_{i}^{2}+\partial_{\mathbf{n}} \kappa\right) F=\kappa, \\
& \phi_{t}+F|\nabla \phi|=0 .
\end{aligned}
$$


The level-set equation (36b) propagates each individual level-set of $\phi$ with speed given by $F$ independently of the other level-sets. Thus, the directional derivative $\partial_{\mathbf{n}} \kappa$ constitutes the only coupling between level-sets in the system (36).

\subsection{Relation to Flow with Constant Speed}

To obtain more inside in the qualitative behavior of the system (26) we consider the special situation $\phi=b_{\Gamma}$ where $b_{\Gamma}$ is the signed distance function to the set $\Gamma=\partial \Omega$ with a smooth bounded open set $\Omega$. In this case, we have $\mathbf{n}^{t}=\nabla b_{\Gamma}, \kappa=\Delta b_{\Gamma}, D \mathbf{n}=D^{2} b_{\Gamma}, D \mathbf{n} \cdot \mathbf{n}=\left(\mathbf{n}^{t} \cdot D \mathbf{n}\right)^{t}=0$, and $|\nabla \phi|=\left|\nabla b_{\Gamma}\right|=1$. Thus, equation (35) reduced to

$$
-\Delta_{\Gamma} \psi+\alpha \psi=\kappa .
$$

on $\Gamma$.

Specifically, for the 2-dimensional situation $n=2$, we consider the closed level curve $\Gamma$ which we write in parametrized form $\Gamma=\{\mathbf{x}(s): s \in[0, L]\}$ with $s$ denoting arc-length and $\mathbf{x}(0)=\mathbf{x}(L)$. Let us set $\alpha=0$ for the moment. Equation (37) can then be written as

$$
-\psi^{\prime \prime}(s)=\kappa(s)
$$

for $s \in[0, L]$ where we set $\psi(s)=\psi(\mathbf{x}(s))$. Integrating with respect to $s$ gives

$$
-\int_{0}^{L} \psi^{\prime \prime}(s) d s=-\psi^{\prime}(L)+\psi^{\prime}(0)=0
$$

for the left-hand side whereas the right-hand side gives

$$
\int_{0}^{L} \kappa(s) d s=\int_{0}^{L} \theta^{\prime}(s) d s=2 \pi
$$

where $\theta$ is the angle between the tangential direction along $\Gamma$ and a fixed reference direction. Therefore, (37) is not solvable for $\alpha=0$. For different choices $\alpha>0$ the solution to (37) with an oscillating right-hand side is shown in Figure 1. The first graphic shows the curvature $\kappa$ along the curve $\Gamma$. The remaining five graphs show the solution to $-\psi^{\prime \prime}+\alpha \psi=\kappa$ for $\alpha=10^{-1}, 10^{-2}, 10^{-3}, 10^{-4}, 10^{-5}$. We plotted the graphs for the different $\alpha$-s of different scales. More precisely, the graphics are scaled such that each $\psi$ is shown in the range between $80 \%$ and $130 \%$ of the average function value. Using the same scale for all graphics would not be useful since the magnitude of the $\phi$ grows approximately like $1 / \alpha$. A variable scaling, however, allows to compare the amount of relative variation, i.e. variation divided by the average, in $\psi$ for different $\alpha$ values. Since $\phi$ acts as an update direction and scaling in $\phi$ can always be compensated by the corresponding reciprocal scaling in the 

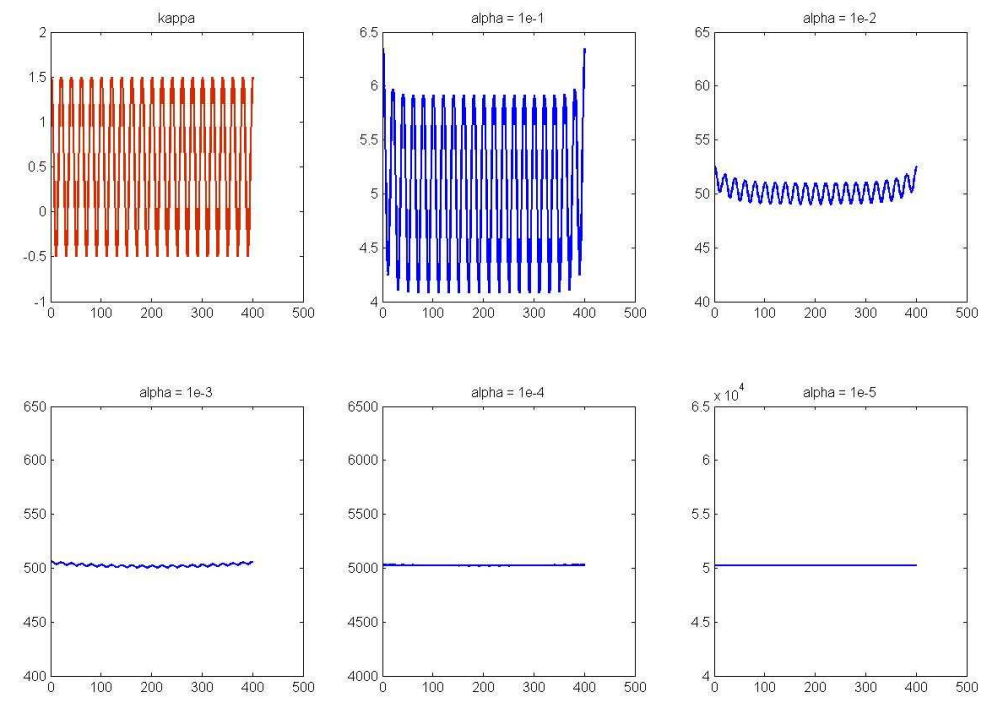

Fig. 1. Behavior of the solution to $-\psi^{\prime \prime}+\alpha \psi=\kappa$ for vanishing $\alpha$ with oscillating right-hand side.

size of the time-step, the graphical representation in Figure 1 is appropriate. It can be seen that $\psi$ becomes approximately constant for small $\alpha$ in the sense that the oscillations become insignificant compared to the overall value of $\psi$. For large $\alpha$ the function $\psi$ is a scaled version of $\kappa$.

The results of the numerical investigation shown in Figure 1 seem to indicate that the Newton-type flow approximates movement by constant normal speed

$$
\frac{\partial \Gamma}{\partial t}=c \mathbf{n}
$$

or, in level-set formulation,

$$
\phi_{t}+c|\nabla \phi|=0 .
$$

The following calculation, however, shows that the constant $c$ depends on the length of the level-set $\Gamma$. We consider a parametrized level-set of $\phi$ given by $\Gamma=\left\{\left(x_{1}(s), x_{2}(s)\right): s \in[0, L]\right\}$ in two space dimensions. Integrating the leftand and right-hand sides of (37) along $\Gamma$ gives

$$
\int_{0}^{L} \psi\left(x_{1}(s), x_{2}(s)\right) d s=\bar{\psi} L=\frac{2 \pi}{\alpha}
$$

i.e. the average speed 


$$
\bar{\psi}=\frac{1}{L} \int_{0}^{L} \psi\left(x_{1}(s), x_{2}(s)\right) d s=\frac{2 \pi}{\alpha L}
$$

of each level set depends on the overall length of the level set. Thus, we can expect to get a geometric evolution equation of the form

$$
\frac{\partial \Gamma}{\partial t}=c(|\Gamma|) \mathbf{n}
$$

with $c(|\Gamma|) \rightarrow \infty$ as $|\Gamma| \rightarrow 0$ in the limit $\alpha \rightarrow 0$. Note that short level sets move (and vanish) faster. On the other hand, large-scale structures (i.e. levelsets with large area) are persistent over longer time-intervals. It is obvious that, for level sets consisting of multiple connected components, the above discussion must be done separately for each connected component. The above discussion holds under the assumption that $\phi=b_{\Gamma}$. Thus, the flow (26) has the described qualitative behavior for small times if the initial level-set function $\phi_{0}$ is a signed distance function. This property, however, is not maintained during the propagation of $\phi$, and we see a different qualitative behavior if the evolution time is sufficiently large.

\subsection{Is this Approach Trivial?}

Let us consider an analogous approach where we replace (11) by the similar functional

$$
K(\phi)=\int_{\mathbb{R}^{n}}|\nabla \phi|^{2} d \mathbf{x}
$$

Obviously

$$
K^{\prime}(\phi) \psi=\frac{1}{2} \int_{\mathbb{R}^{n}}\langle\nabla \phi, \nabla \psi\rangle d \mathbf{x} \quad \text { and } \quad K^{\prime \prime}(\phi)(\psi, \psi)=\frac{1}{2} \int_{\mathbb{R}^{n}}\langle\nabla \psi, \nabla \psi\rangle d \mathbf{x}
$$

The optimality system for the determination of a Newton-type descent direction — analogous to (25) — then reads as

$$
\frac{1}{2} \int_{\mathbb{R}^{n}}\langle\nabla \phi, \nabla \eta\rangle d \mathbf{x}+\frac{\lambda}{4} \int_{\mathbb{R}^{n}}\langle\nabla \psi, \nabla \eta\rangle d \mathbf{x}=0
$$

for all test functions $\eta$. This equation obviously has a solution $\psi=c \phi$ leading to the rather trivial flow equation

$$
\phi_{t}=-\phi
$$

which is of zeroth-order in $\mathbf{x}$. The solution $\phi(\mathbf{x}, t)=e^{-t} \phi_{0}(\mathbf{x})$ clearly diminishes the value of the functional (39).

The situation is not as simple for the non-quadratic functional $\tilde{J}$. Let us consider the situation $\phi=b_{\Gamma}$ and the formulation (35) with $\alpha=0$. We get

$$
-\Delta_{\Gamma} \psi=\Delta \phi
$$


or

$$
-\operatorname{div}_{\Gamma}\left(\nabla_{\Gamma} \psi\right)=\operatorname{div}_{\Gamma}(\nabla \phi),
$$

where $\operatorname{div}_{\Gamma} \mathbf{v}=\operatorname{div} \mathbf{v}-\mathbf{n}^{t} \cdot D \mathbf{v} \cdot \mathbf{n}$ is the tangential divergence of a vector field $\mathbf{v}$. This equation looks like we can again set $\psi=-\phi$ to obtain a solution. This is, however, not the case since $\nabla \phi$ is orthogonal to $\Gamma$, and therefore $\nabla_{\Gamma} \phi=0$.

\subsection{Relation to inverse scale space methods}

Recently [4] the following system of equations was introduced

$$
\begin{aligned}
p_{t} & =f-\phi \\
p & =-\operatorname{div}\left(\frac{\nabla \phi}{|\nabla \phi|}\right)
\end{aligned}
$$

for the smoothing of a noisy image $f$. The approach was called an inverse scale space method due to the property that — starting with zero - large features are restored first and fine structures and noise appear late in the evolution of the system (40). The approach can be seen as the continuous limit of an iterated Tikhonov regularization (or proximal point) algorithm for quadratic cost functionals, or as a continuous limit of Bregman iterations for the nonquadratic case as in (40). See also [23] for an analogous approach for linear problems. We shall show that (40) is closely related to the Newton-flow (26) although the starting point of the investigations in [4] is quite different from the approach presented here. To this aim, we write (40) in weak form

$$
-\int_{0}^{\infty} \int_{\mathbb{R}^{n}} p \tilde{\eta}_{t} d \mathbf{x} d t=\int_{0}^{\infty} \int_{\mathbb{R}^{n}}(f-\phi) \tilde{\eta} d \mathbf{x} d t
$$

for all test functions $\tilde{\eta} \in \mathcal{D}\left((0, \infty) \times \mathbb{R}^{n}\right)$, and

$$
\int_{\mathbb{R}^{n}} p \eta d \mathbf{x}=\int_{\mathbb{R}^{n}} \frac{1}{|\nabla \phi|}\langle\nabla \phi, \nabla \eta\rangle d \mathbf{x}
$$

for all $\eta \in \mathcal{D}\left(\mathbb{R}^{n}\right)$. Setting $\eta=\tilde{\eta}_{t}$ in (42) and integrating with respect to $t$ gives

$$
\int_{0}^{\infty} \int_{\mathbb{R}^{n}} p \tilde{\eta}_{t} d \mathbf{x} d t=\int_{0}^{\infty} \int_{\mathbb{R}^{n}} \frac{1}{|\nabla \phi|}\left\langle\nabla \phi, \nabla \tilde{\eta}_{t}\right\rangle d \mathbf{x} d t
$$

for all $\tilde{\eta} \in \mathcal{D}\left((0, \infty) \times \mathbb{R}^{n}\right)$. Partial integration of the right-hand side of (43) with respect to $t$ together with (41) yields

$$
\begin{aligned}
\int_{0}^{\infty} \int_{\mathbb{R}^{n}}\left[\frac { 1 } { | \nabla \phi | } \left(\left\langle\nabla \phi_{t}, \nabla \tilde{\eta}\right\rangle-\left\langle\frac{\nabla \phi}{|\nabla \phi|}, \nabla \phi_{t}\right\rangle\right.\right. & \left.\left.\left\langle\frac{\nabla \phi}{|\nabla \phi|}, \nabla \tilde{\eta}\right\rangle\right)\right] d \mathbf{x} d t \\
& =\int_{0}^{\infty} \int_{\mathbb{R}^{n}}(f-\phi) \tilde{\eta} d \mathbf{x} d t
\end{aligned}
$$


for all test functions $\tilde{\eta} \in \mathcal{D}\left((0, \infty) \times \mathbb{R}^{n}\right)$. Setting $\tilde{\eta}(\mathbf{x}, t)=\eta(\mathbf{x}) \zeta(t)$ and letting $\zeta$ vary throughout $\mathcal{D}((0, \infty))$, we conclude from (44) that

$$
\int_{\mathbb{R}^{n}}\left[\frac{1}{|\nabla \phi|}\left(\left\langle\nabla \phi_{t}, \nabla \eta\right\rangle-\left\langle\frac{\nabla \phi}{|\nabla \phi|}, \nabla \phi_{t}\right\rangle\left\langle\frac{\nabla \phi}{|\nabla \phi|}, \nabla \eta\right\rangle\right)\right] d \mathbf{x}=\int_{\mathbb{R}^{n}}(f-\phi) \eta d \mathbf{x}
$$

holds for all $\eta \in \mathcal{D}\left(\mathbb{R}^{n}\right)$. If we set $\psi=\phi_{t}$, we obtain the coupled system

$$
\begin{aligned}
& \int_{\mathbb{R}^{n}}\left[\frac{1}{|\nabla \phi|}\left(\langle\nabla \psi, \nabla \eta\rangle-\left\langle\frac{\nabla \phi}{|\nabla \phi|}, \nabla \psi\right\rangle\left\langle\frac{\nabla \phi}{|\nabla \phi|}, \nabla \eta\right\rangle\right)\right] d \mathbf{x}=\int_{\mathbb{R}^{n}}(f-\phi) \eta d \mathbf{x} \\
& \phi_{t}=\psi
\end{aligned}
$$

for all test functions $\eta \in \mathcal{D}\left(\mathbb{R}^{n}\right)$. Thus, the system (46) is very similar to (26). The difference between the two is that the right hand side (the source term) in (26a) represents curvature whereas the right-hand side in (46a) represents the data fit $f-\phi$. Moreover, the zero-order regularization term on the lefthand side in (26a) is not present in (46a). The usual initial value for the flow (46) is $\phi_{0}=0$. Using this initial value, it is observed that $\phi$ evolves towards that given data function $f$ with large features and texture appearing first and random noise appearing only in the late stages of the evolution.

We can also go in the reverse direction and rewrite the weak Newton flow (26) in a form similar to (40). We set again $p=-\operatorname{div}\left(\frac{\nabla \phi}{|\nabla \phi|}\right)$. Then $p$ appears on the right-hand side of (30) and

$$
p_{t}=-\operatorname{div}\left(\frac{1}{|\nabla \phi|}\left(\nabla \phi_{t}-\left\langle\frac{\nabla \phi}{|\nabla \phi|}, \nabla \phi_{t}\right\rangle \frac{\nabla \phi}{|\nabla \phi|}\right)\right)
$$

appears in the left-hand side of (30) if $\psi$ is replaced by $\phi_{t}$. Thus, we obtain

$$
\begin{aligned}
& p_{t}+\alpha \frac{\phi_{t}}{|\nabla \phi|}=-p \\
& p=-\operatorname{div}\left(\frac{\nabla \phi}{|\nabla \phi|}\right)
\end{aligned}
$$

for a formulation of the Newton flow which is analogous to (40).

\subsection{Boundary conditions}

Any numerical solution of (33) requires to replace $\mathbb{R}^{n}$ by a bounded open domain $D$ as domain of definition. In this case, boundary conditions for $\psi$ have to be specified. We use the weak formulation

$$
\begin{aligned}
\int_{D} \frac{1}{|\nabla \phi|}\left(\langle\nabla \psi, \nabla \eta\rangle-\left\langle\frac{\nabla \phi}{|\nabla \phi|}, \nabla \psi\right\rangle\left\langle\frac{\nabla \phi}{|\nabla \phi|}, \nabla \eta\right\rangle\right. & +\alpha \psi \eta) d \mathbf{x} \\
= & \int_{D} \operatorname{div}\left(\frac{\nabla \phi}{|\nabla \phi|}\right) \eta d \mathbf{x}
\end{aligned}
$$


for all test functions $\eta \in \mathcal{D}\left(\mathbb{R}^{n}\right)$.

Let $\boldsymbol{\nu}$ denote the outer unit normal to the set $D$. Application of Green's formula shows that the boundary condition is given in strong form as

$$
\left\langle\nabla_{\Gamma} \psi, \boldsymbol{\nu}\right\rangle=0
$$

on $\partial D$. Here $\nabla_{\Gamma}$ denotes the tangential derivative with respect to $\Gamma$. If $\boldsymbol{\nu}$ is not normal to $\Gamma$, equation (48) is in fact a homogenous Neumann-type boundary condition on $\Gamma \cap \partial D$ for the intrinsic equation (35).

Note that we have integrated the right hand side of $(26 \mathrm{a})$ by parts to obtain (47) but we have omitted the boundary term $-\int_{\partial D}\langle\mathbf{n}, \boldsymbol{\nu}\rangle \eta d S$. With this modification, (47) is not derived from the cost functional

$$
\int_{D}|\nabla \phi| d \mathbf{x}
$$

along the same (variational) reasoning as (26). If this was done, we end up with a non-homogeneous boundary condition

$$
\left\langle\nabla_{\Gamma} \psi, \boldsymbol{\nu}\right\rangle=-\langle\mathbf{n}, \boldsymbol{\nu}\rangle|\nabla \phi| .
$$

This type of boundary condition takes into account the effect of shrinking the area of level sets within $D$ by pushing them out of $D$ across $\partial D$. We choose the boundary condition (48) to avoid this type of influence of the boundary on the evolution of level-sets. Note also that the non-homogeneous boundary condition (50) introduces singularities at points $\mathbf{x} \in \Gamma \cap \partial D$ where $\boldsymbol{\nu}= \pm \mathbf{n}$. At these points the right-hand side of (50) is zero and the left-hand side is in general different from zero.

\section{Numerical Examples}

Our numerical examples exemplify the behavior of the system (26), i.e. we use the weak formulation of the Newton-type flow. For fixed $\phi$, the degenerate elliptic equation (26a) is solved for $\psi$ using bilinear tensor splines for the discretization of $\psi$. The singularity at $\nabla \phi=0$ is treated replacing $|\nabla \phi|$ by $\left(|\nabla \phi|^{2}+\epsilon^{2}\right)^{\frac{1}{2}}$ whenever $\nabla \phi$ occurs in the denominator. It turned out to be better to regularize not only $|\nabla \phi|$ in the denominator, but to replace $\mathbf{n}=\frac{\nabla \phi}{|\nabla \phi|}$ by $\frac{\nabla \phi+\mathbf{e}^{t}}{\left|\nabla \phi+\mathbf{e}^{t}\right|}$ with a small vector $\mathbf{e}$ which pushes $\nabla \phi$ away from zero at all occurrences of $\mathbf{n}$ in (26a). Most of the examples are carried out with the geometric $L^{2}$-regularization (21). Only at the end of this section we make a comparison between the formulations (21) and (22).

We begin the experimental study of properties of the flow (26) by comparing the propagations of a noise-free, non-convex level curve for different values of the regularization parameter $\alpha$. By gradually increasing $\alpha$, the characteristic of the flow changes from a global shrinking of level-sets with constant 
speed to a local shrinking of level-sets by diminishing the curvature locally. We also mention that the admissible step-size of the flow decreases with increasing $\alpha$, i.e. the more the flow resembles mean-curvature motion. The initial level-set function $\phi_{0}$ is chosen as the signed distance function of the initial curve. The plots in Figure 2 show the zero level-sets of the level set function $\phi$ at every $k^{\text {th }}$ iteration of the time-stepping algorithm ( $k$ between 20 and 80 for the individual choices of $\alpha$ ), where the time-step is controlled by a fixed CFL-like criterion. The condensation of level-sets within certain time intervals is explained by the development of blocky structures in the level-set function $\phi$. If a situation is reached, where blocky structures with sharp edges have occurred, the propagation is decelerated mainly due to the reduction of the admissible time-step which depends on $|\nabla \phi|$. In sections, where this influence of the time-step reduction is not yet active, an acceleration of the speed of propagation for smaller level-sets can be observed. This uneven spacing of level-lines for consecutive iteration numbers constitutes a structural difference between our flow (for small $\alpha$ ) and flow with constant speed (i.e. construction of the signed distance function of an initial curve). Note that, for small $\alpha$, the initially connected level set splits into two parts. This behavior is impossible for classical mean curvature flow. (See $[21,22]$.)
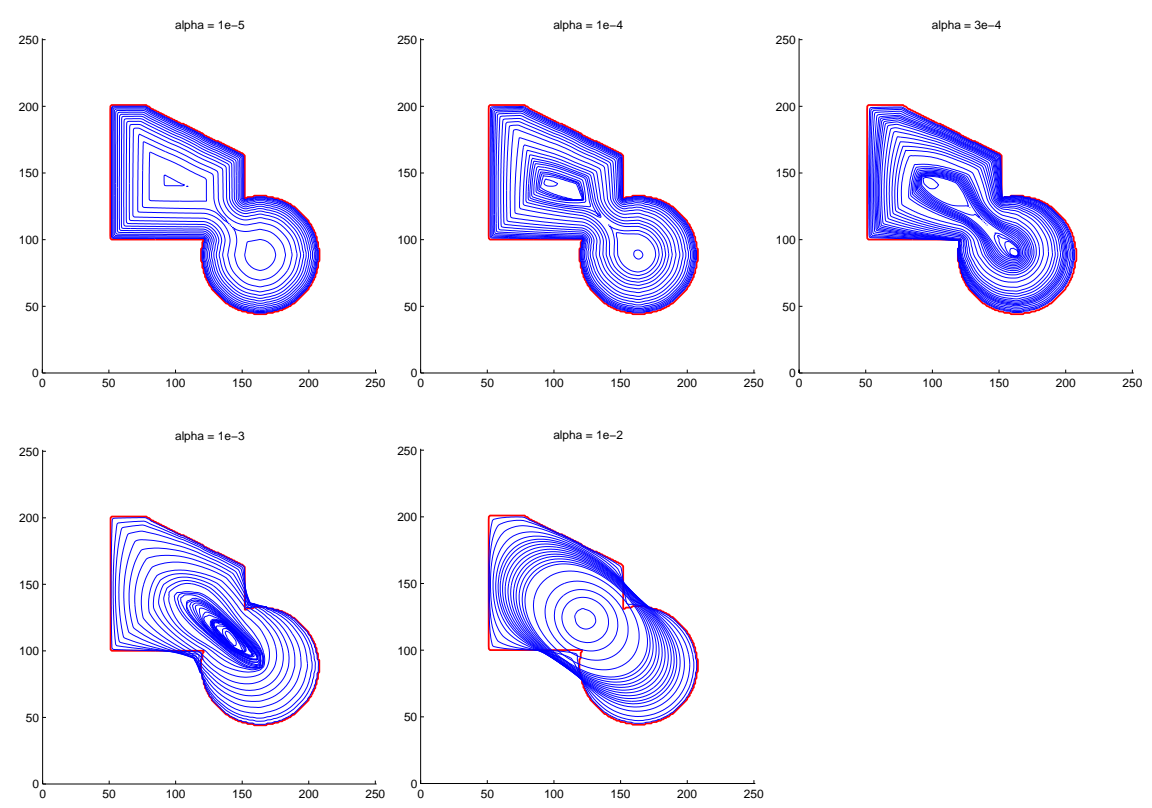

Fig. 2. Behavior of the Newton-type flow for $\alpha=10^{-5}, 10^{-4}, 3 \cdot 10^{-3}, 10^{-3}, 10^{-2}$. Gradual interpolation between constant speed and mean curvature flow. 
The next Figure 3 shows that the average speed of different level sets depends on their respective size with smaller level-sets moving faster. The initial level-sets are drawn in red. In blue the zero-level set (consisting of multiple components of different size) is drawn every 15 time-steps. It can be clearly seen that the propagation accelerates the smaller the components get. We mention, that the situation is such that during the whole propagation the level-set function is still close to a signed-distance function and blocky structures have not yet developed.
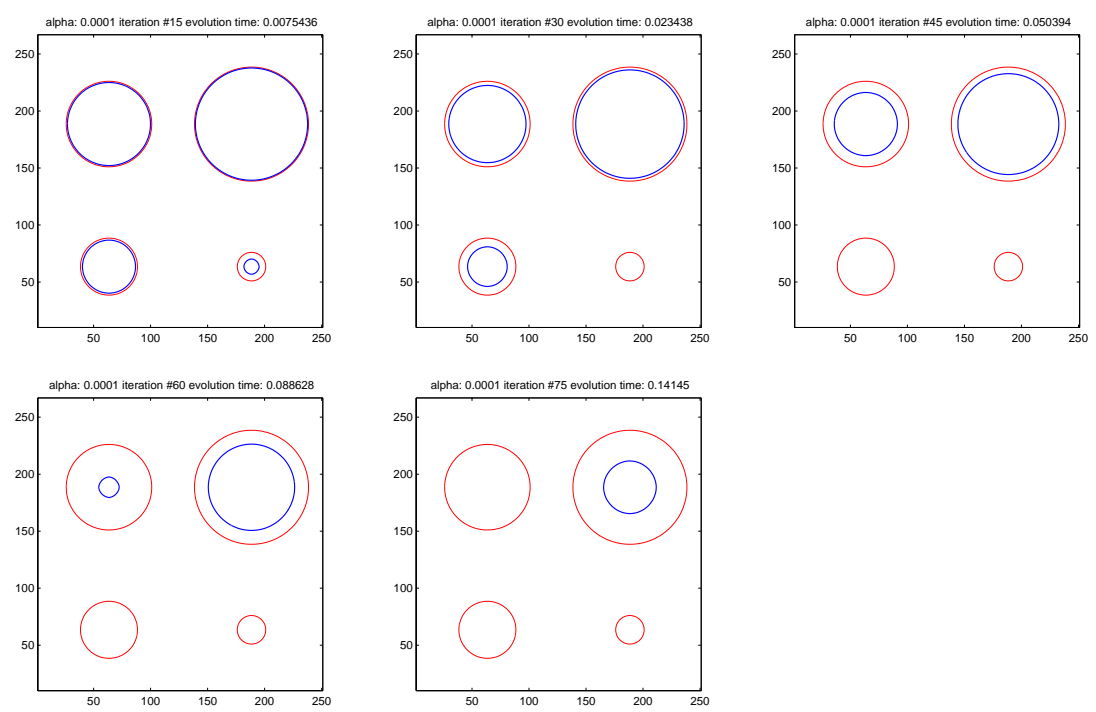

Fig. 3. Behavior of the Newton-type flow for small $\alpha=10^{-4}$ and initial level-sets of different size. Smaller level-sets move faster.

In Figure 4 the behavior of the flow for oscillating initial data is illustrated. We have used the level-set function $\phi$ of the experiments shown in Figure 2 and overlayed it with a highly oscillating perturbation. The experiment is carried out with small $\alpha=10^{-5}$. It is seen that the length of the curve is not reduced by local smoothing but by shrinking the oscillating structure globally.

Figure 5 shows the evolution of a real noise-free image under the Newtontype flow (26). It is seen that smooth structures are flattened out fast, whereas edges and texture are maintained very well. Note that the texture of the feathers and the straw-hat in the Lena image are still maintained (and even enhanced to some extend) when smoother structures as the nose or the structure in the left background have already vanished. Step edges as e.g. the frame of the mirror on the right-hand side of the image are maintained longer than smoother structures but not as long as textured structures as e.g. the 


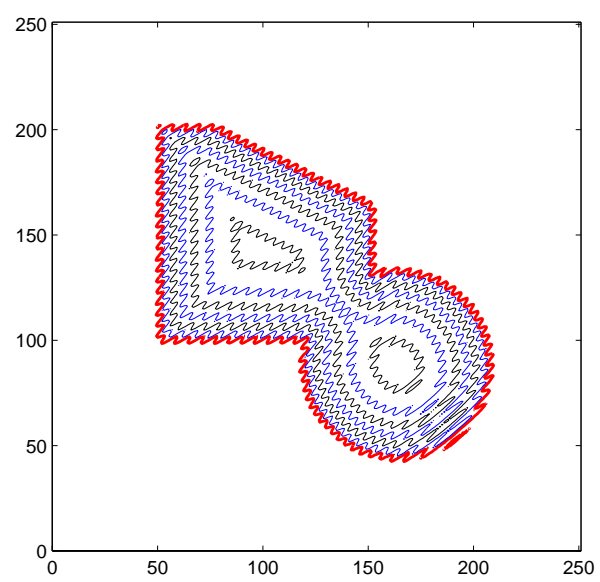

Fig. 4. Newton-type flow of a curve with highly oscillating curvature for $\alpha=10^{-5}$. The length of the curve is reduced globally, not locally.

straw-hat. Note also that the smaller bright structure in the lower left corner vanishes much earlier that the larger structure along the left edge of the image, although their initial brightness, contrast, and sharpness are very much the same. Figure 6 is an enlarged version of two images in Figure 5. The persistent edges and textures are clearly visible. The flow reduces the overall contrast of the images from the initial range of gray values between 0 and 255 to the interval between 75 and 145 at iteration no. 1200. For better visibility the images have been scaled so that minimal intensity appears black and the maximal intensity appears white. Other numerical experiments with noisy images indicate that high frequency noise is removed from a level-set function (i.e. an image) more or less in the same amount as signal is removed. Thus, the Newton-flow - for small alpha - is not well suited for image denoising.

In the last Figure 7 a comparison between regularization by the geometric $L^{2}$-norm (16) and the non-geometric $L^{2}$-norm (18) is shown. The choice $\alpha=$ $10^{-3}$ has been made to find a situation where the regularization term has some influence on the behavior of the flow, but $\alpha$ is not large enough to change the characteristic of the flow strongly towards pure mean curvature or total variation flow. It is seen that the non-geometric version to the left has a certain preference for flat regions and blocky structures, whereas the geometric regularization to the right maintains smooth transitions of gray values slightly better.

\section{Conclusion}

We have introduced a nonlocal flow equation which reduces the area of levelsets of an initial function based on a gradient descent flow with respect to a 

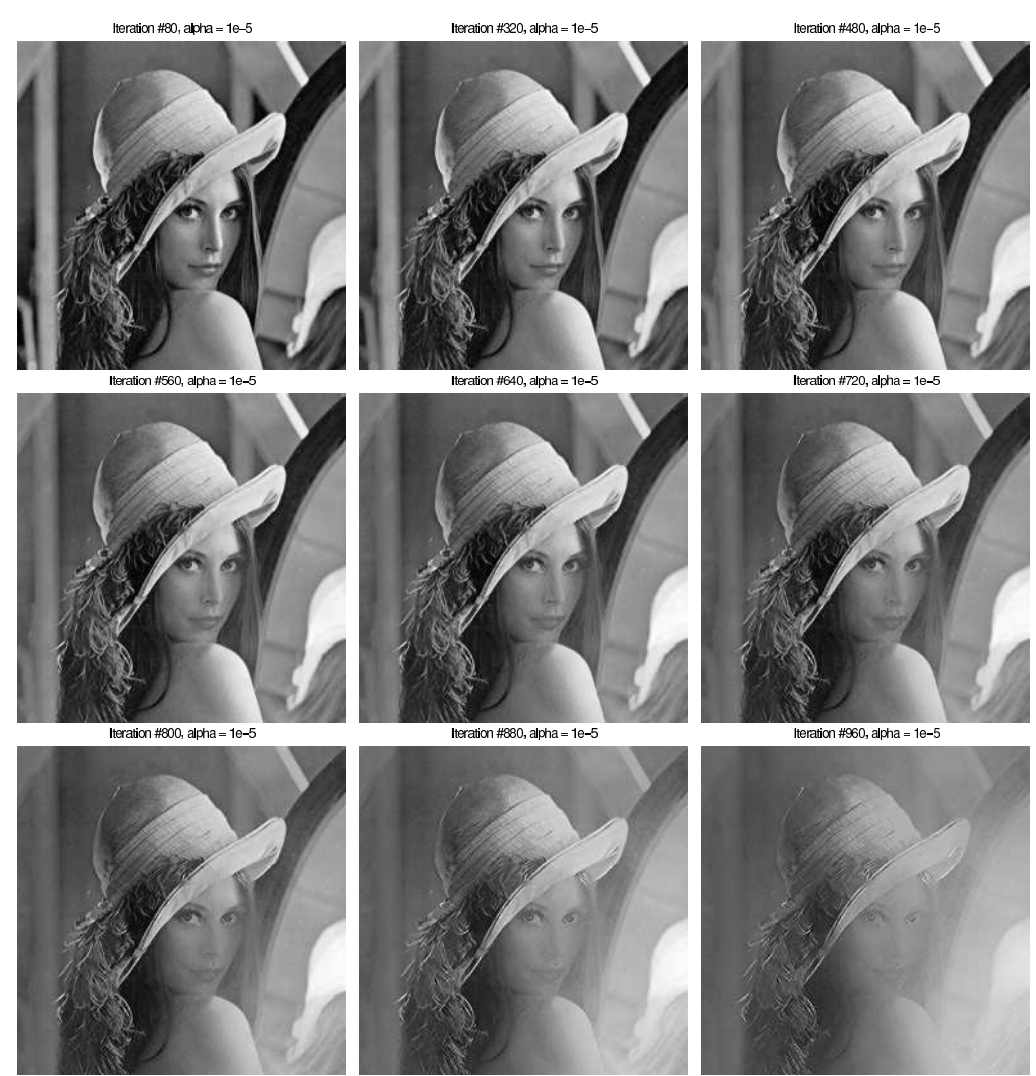

Iteration \#1040, alpha = 1e-5

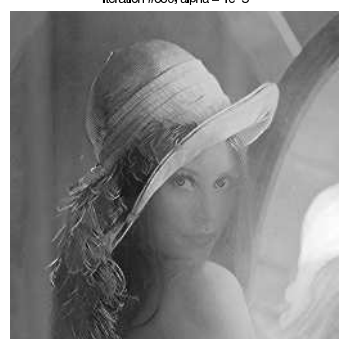

Iteration \#1120, alpha = 10-5
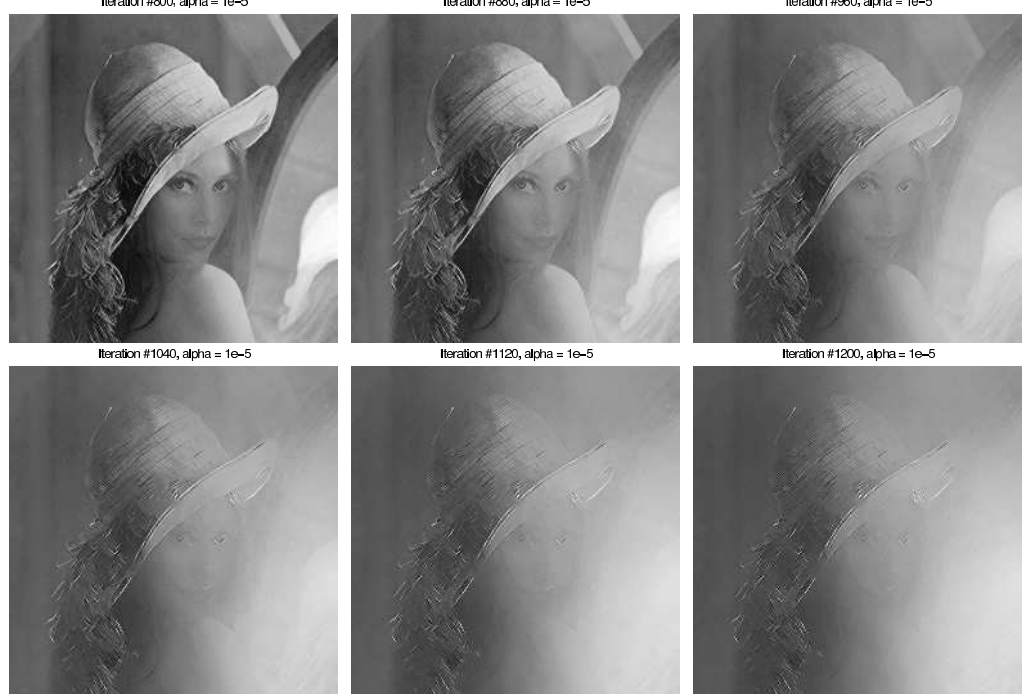

Iteration \#1200, abha = 10-5

Heration \#1280, alpha = 1e-5

Iteration \#1360, alpha = 10-5
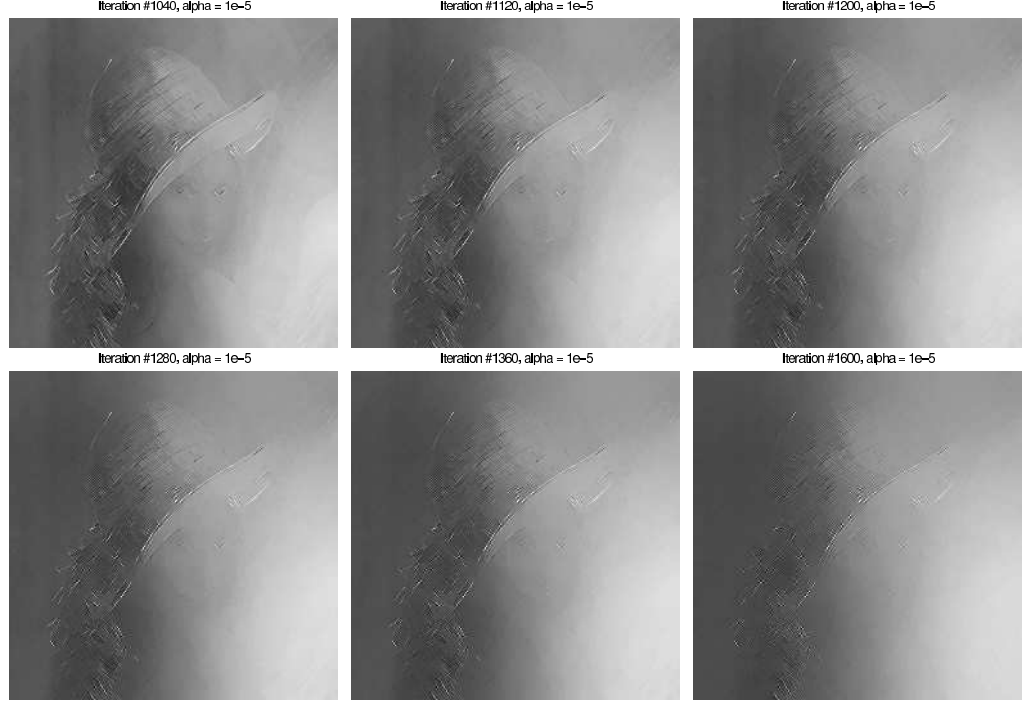

Fig. 5. Newton-type flow for the Lena image. $\alpha=10^{-5}$. Smooth structures are removed, edges and texture are maintained 
Iteration \#800, alpha $=1 \mathrm{e}-5$
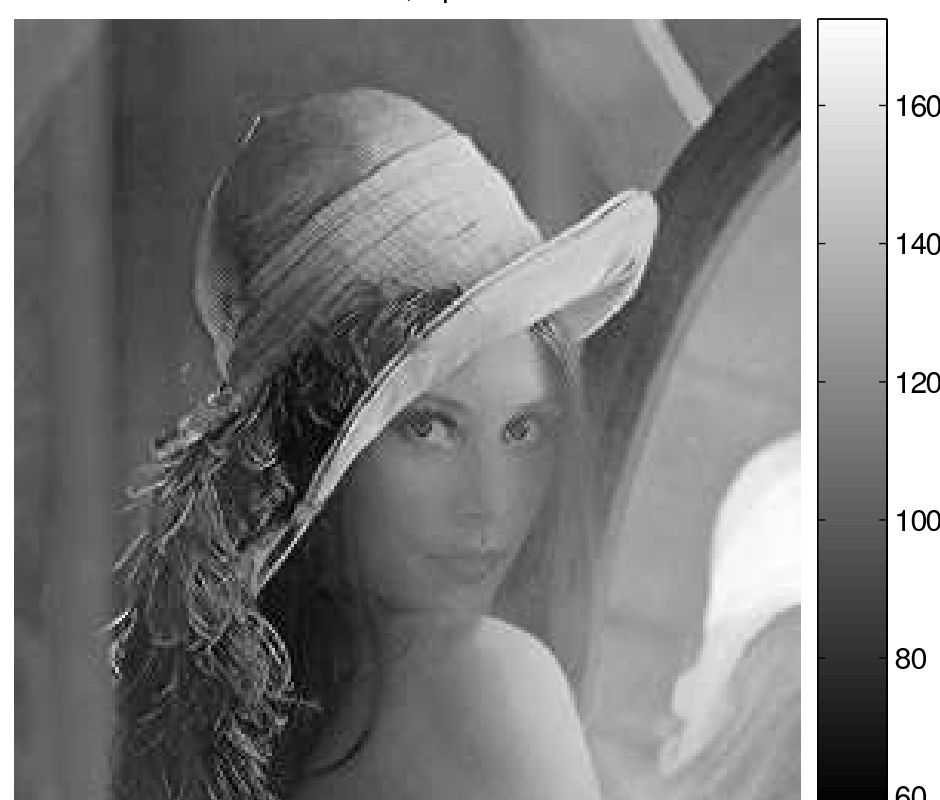

Iteration \#1200, alpha = 1e-5

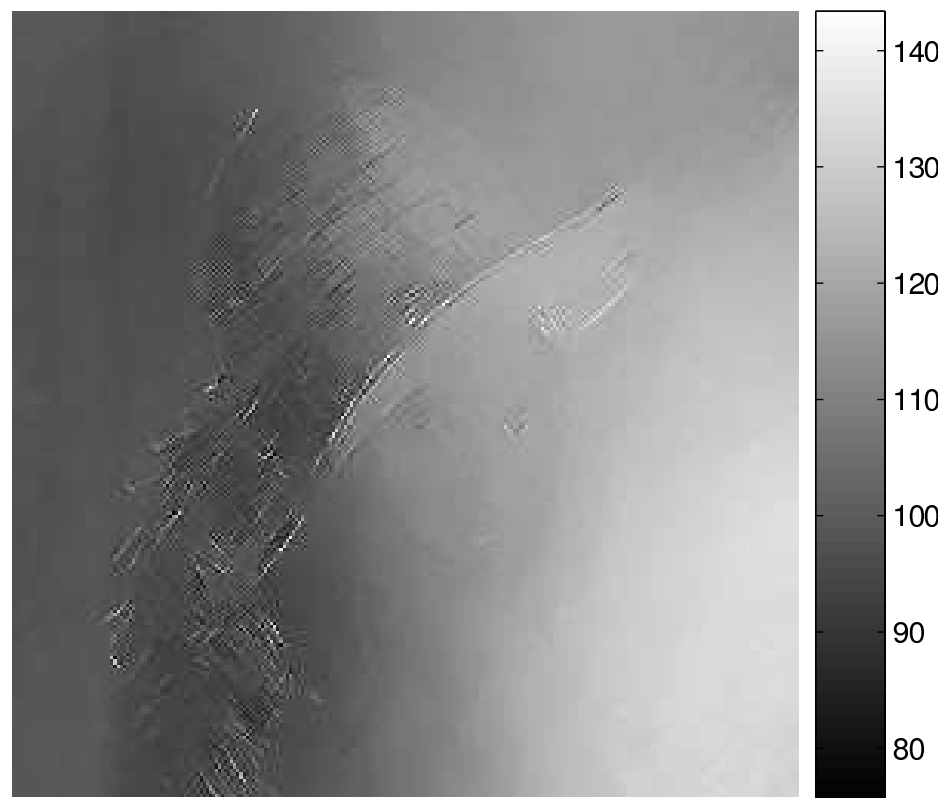

Fig. 6. Magnified Lena image at iterations 800 and 1200. 


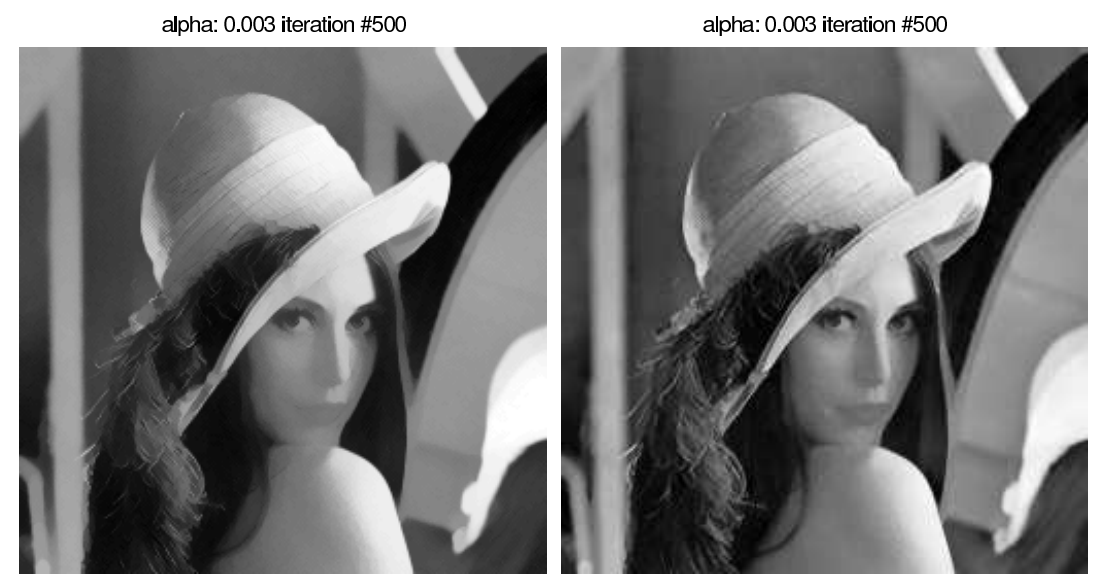

Fig. 7. Comparison between $L^{2}$-regularization (left) and geometric regularization (right) for $\alpha=10^{-3}$.

variable metric constructed from the second derivative of the cost functional. We have investigated geometrical properties of the flow and we have compared our approach with the recently introduced inverse scale space method. Numerical experiments were presented illustrating features of the flow for single propagating level-sets and for images for which all level-sets propagate simultaneously.

The practical applicability of the approach as it stands is probably limited to situations, where small structures are to be removed from an image but large structures should remain untouched. Our approach treads noise as large, wiggled level-sets which are spread over a certain area in space. Such level sets are shrunk very slowly, therefore noise is not remove from the image. The general approach of constructing a Newton-type flow for an arbitrary geometric functional in the level-set context has a variety of potential applications. Expected benefits are speed up of the minimization algorithm and the occurrence of interesting features in the flows due to the availability of non-local information.

\section{References}

1. F. Andreu, C. Ballester, V. Caselles, and J. M. Mazón, Minimizing total variation flow, Differential Integral Equations, 14 (2001), pp. 321-360.

2. G. Aubert, M. Barlaud, O. Faugeras, and S. Jehan-Besson, Image segmentation using active contours: calculus of variations or shape gradients?, SIAM J. Appl. Math., 63 (2003), pp. 2128-2154 (electronic).

3. V. BARBu, Analysis and Control of Nonlinear Infinite Dimensional Systems, vol. 190 of Mathematics in Science and Engineering, Academic Press, Boston, 1993. 
4. M. Burger, G. GilboA, S. Osher, And J. Xu, Nonlinear inverse scale space methods, Communications in Mathematical Sciences, 4 (2006).

5. F. CAO, Geometric curve evolution and image processing, vol. 1805 of Lecture Notes in Mathematics, Springer-Verlag, Berlin, 2003.

6. V. Caselles, R. Kimmel, And G. SAPiro, Geodesic active contours, Int. J. of Computer Vision, 22 (1997), pp. 61-79.

7. A. Chambolle, An algorithm for mean curvature motion, Interfaces and Free Boundaries, 6 (2004), pp. 195-218.

8. Y. G. Chen, Y. Giga, And S. Goto, Uniqueness and existence of viscosity solutions of generalized mean curvature flow equations, J. Differential Geom., 33 (1991), pp. 749-786.

9. M. G. Crandall, H. Ishit, And P.-L. Lions, User's guide to viscosity solutions of second order partial differential equations, Bull. Amer. Math. Soc. (N.S.), 27 (1992), pp. 1-67.

10. K. Deckelnick And G. Dziuk, Mean curvature flow and related topics, in Frontiers in numerical analysis (Durham, 2002), Universitext, Springer, Berlin, 2003, pp. 63-108.

11. - Numerical approximation of mean curvature flow of graphs and level sets, in Mathematical aspects of evolving interfaces (Funchal, 2000), vol. 1812 of Lecture Notes in Math., Springer, Berlin, 2003, pp. 53-87.

12. K. Deckelnick, G. Dziuk, And C. M. Elliott, Computation of geometric partial differential equations and mean curvature flow, Acta Numer., 14 (2005), pp. 139-232.

13. M. C. Delfour And J.-P. ZolÉsio, Shapes and geometries, Society for Industrial and Applied Mathematics (SIAM), Philadelphia, PA, 2001. Analysis, differential calculus, and optimization.

14. D. C. Dobson And C. R. Vogel, Convergence of an iterative method for total variation denoising, SIAM J. Numer. Anal., 34 (1997), pp. 1779-1791.

15. M. Droske And M. Rumpf, A level set formulation for Willmore flow, Interfaces and Free Boundaries, 6 (2004), pp. 361-378.

16. L. C. Evans And R. F. GARIEPy, Measure theory and fine properties of functions, CRC Press, Boca Raton, FL, 1992.

17. L. C. Evans AND J. Spruck, Motion of level sets by mean curvature. I, J. Differential Geom., 33 (1991), pp. 635-681.

18. - Motion of level sets by mean curvature. II, Trans. Amer. Math. Soc., 330 (1992), pp. 321-332.

19. _ , Motion of level sets by mean curvature. III, J. Geom. Anal., 2 (1992), pp. 121-150.

20. — Motion of level sets by mean curvature. IV, J. Geom. Anal., 5 (1995), pp. $77-114$.

21. M. Gage And R. S. Hamilton, The heat equation shrinking convex plane curves, J. Differential Geom., 23 (1986), pp. 69-96.

22. M. A. GRAYSON, The heat equation shrinks embedded plane curves to round points, J. Differential Geom., 26 (1987), pp. 285-314.

23. C. Groetsch And O. Scherzer, Non-stationary iterated Tikhonov-Morozov method and third-order differential equations for the evaluation of unbounded operators., Math. Methods Appl. Sci., 23 (2000), pp. 1287-1300.

24. S. Kichenassamy, A. Kumar, P. Olver, A. Tannenbaum, and A. Yezzi, Conformal curvature flows: from phase transitions to active vision, Arch. Rational Mech. Anal., 134 (1996), pp. 275-301. 
25. W. KüHnel, Differential geometry, vol. 16 of Student Mathematical Library, American Mathematical Society, Providence, RI, 2002. Curves - surfacesmanifolds, Translated from the 1999 German original by Bruce Hunt.

26. J. Nocedal and S. J. Wright, Numerical optimization, Springer Series in Operations Research, Springer-Verlag, New York, 1999.

27. S. Osher And J. A. SethiAn, Fronts propagating with curvature-dependent speed: algorithms based on Hamilton-Jacobi formulations, J. Comput. Phys., 79 (1988), pp. 12-49.

28. L. Rudin, S. Osher, And E. FAtemi, Nonlinear total variation based noise removal algorithm, Physica D, 60 (1992), pp. 259-268.

29. J. SokoŁOWski AND J.-P. ZoLÉSIO, Introduction to shape optimization, Springer-Verlag, Berlin, 1992. Shape sensitivity analysis.

30. J. E. Solem And N. C. OvergaARd, A geometric formulation of gradient descent for variational problems with moving surfaces., in Scale space and PDE methods in computer vision. 5th international conference, Scale-Space 2005, Hofgeismar, Germany, April 7-9, 2005. Proceedings., R. Kimmel, N. Sochen, and J. Weickert, eds., Lecture Notes in Computer Science 3459, Springer, 2005, pp. $419-430$.

31. C. R. Vogel And M. E. Oman, Iterative methods for total variation denoising, SIAM J. Sci. Comput., 17 (1996), pp. 227-238. Special issue on iterative methods in numerical linear algebra (Breckenridge, CO, 1994).

32. A. Yezzi, S. Kichenassamy, A. Kumar, P. Olver, and A. Tannenbaum, A geometric snake model for segmentation of medical imagery, IEEE Trans. Med. Imaging, 16 (1997), pp. 199-209. 